\title{
RANDOM DIOPHANTINE EQUATIONS
}

\author{
BJORN POONEN AND JOSÉ FELIPE VOLOCH
}

(With appendices by Jean-Louis Colliot-Thélène And Nicholas M. Katz)

\section{INTRODUCTION}

The main result of this paper is that, in a precise sense, a positive proportion of all hypersurfaces in $\mathbf{P}^{n}$ of degree $d$ defined over $\mathbf{Q}$ are everywhere locally solvable, provided that $n, d \geq 2$ and $(n, d) \neq(2,2)$. This result is motivated by a conjecture discussed in detail below about the proportion of hypersurfaces as above that are globally solvable, i.e., have a rational point.

\section{A CONJECTURE}

Fix $n, d \geq 2$. Let $\mathbf{Z}\left[x_{0}, \ldots, x_{n}\right]_{d}$ denote the set of homogeneous polynomials in $\mathbf{Z}\left[x_{0}, \ldots, x_{n}\right]$ of degree $d$. Let $m=\left(\begin{array}{c}n+d \\ d\end{array}\right)$ denote the number of monomials in $x_{0}, \ldots, x_{n}$ of degree $d$. Define the height $h(f)$ of $f \in \mathbf{Z}\left[x_{0}, \ldots, x_{n}\right]_{d}$ as the maximum of the absolute values of the coefficients of $f$. Let $M_{\mathbf{Q}}$ be the set of places of $\mathbf{Q}$, and let $\mathbf{Q}_{v}$ be the completion of $\mathbf{Q}$ at the place $v$. Define

$$
\begin{aligned}
N_{\text {tot }}(H) & =\#\left\{f \in \mathbf{Z}\left[x_{0}, \ldots, x_{n}\right]_{d}: h(f) \leq H\right\}=(2\lfloor H\rfloor+1)^{m}, \\
N(H) & =\#\left\{f \in \mathbf{Z}\left[x_{0}, \ldots, x_{n}\right]_{d}: h(f) \leq H, \text { and } \exists x \in \mathbf{Z}^{n+1} \backslash\{0\} \text { with } f(x)=0\right\}, \\
N_{\text {loc }}(H) & =\#\left\{f \in \mathbf{Z}\left[x_{0}, \ldots, x_{n}\right]_{d}: h(f) \leq H, \text { and } \forall v \in M_{\mathbf{Q}}, \exists x \in \mathbf{Q}_{v}^{n+1} \backslash\{0\} \text { with } f(x)=0\right\} .
\end{aligned}
$$

The limit of $N(H) / N_{\text {tot }}(H)$ as $H \rightarrow \infty$, if it exists, will be called the proportion of globally solvable hypersurfaces. Similarly, the limit of $N_{\text {loc }}(H) / N_{\text {tot }}(H)$ will be called the proportion of locally solvable hypersurfaces.

\section{Remark 2.1.}

(1) Restricting the set of $f^{\prime}$ 's to those such that $f=0$ defines a smooth geometrically integral hypersurface in $\mathbf{P}^{n}$ does not change the values of these limits, since the $f^{\prime}$ 's that violate these conditions correspond to integer points on a Zariski closed subset of positive codimension in some affine space.

(2) A standard argument involving Möbius inversion shows that the values of the limits do not change if in all our counts we restrict to $f$ 's whose coefficients are coprime.

(3) Everything we do in this paper over $\mathbf{Q}$ could be generalized to number fields without difficulty. We chose to work over $\mathbf{Q}$ to keep statements and proofs simple.

\section{Conjecture 2.2.}

Date: March 1, 2003.

2000 Mathematics Subject Classification. Primary 11D72; Secondary 11G35, 14G25.

Key words and phrases. Diophantine equation, Hasse principle, Brauer-Manin obstruction, hypersurface, complete intersection, Weak Lefschetz Theorem, cubic surface.

The first author was partially supported by a Packard Fellowship. 
(i) If $d>n+1$, then $N(H) / N_{\text {tot }}(H) \rightarrow 0$.

(ii) If $d<n+1$ and $(d, n) \neq(2,2)$, then $N(H) / N_{\text {tot }}(H) \rightarrow c$ for some real $c>0$. Moreover, $c=\prod_{v \in M_{\mathbf{Q}}} c_{v}$, where $c_{v}$ is the proportion of polynomials in $\mathbf{Z}\left[x_{0}, \ldots, x_{n}\right]_{d}$ with a nontrivial zero over $\mathbf{Q}_{v}$.

In the case $d=n+1$, we do not know what to expect. As a special case, if you write down a plane cubic, how likely is it to have a rational point?

Remark 2.3. Each local proportion $c_{v}$ exists, since if we define $\mathbf{Z}_{v}=\left\{x \in \mathbf{Q}_{v}:|x|_{v} \leq 1\right\}$ and normalize Haar measure (Lebesgue measure if $v=\infty$ ) on the space $\mathbf{Z}_{v}^{m}$ parametrizing homogeneous polynomials of degree $d$ in $x_{0}, \ldots, x_{n}$ with coefficients in $\mathbf{Z}_{v}$, then $c_{v}$ is the measure of the $v$-adically closed subset of $\mathbf{Z}_{v}^{m}$ corresponding to homogeneous polynomials with a nontrivial zero over $\mathbf{Q}_{v}$.

\section{Motivation And EVIDEnCE}

To motivate the first part of the conjecture, consider the set of $f \in \mathbf{Z}\left[x_{0}, \ldots, x_{n}\right]_{d}$ of height at most $H$ having a given zero $a \in \mathbf{Z}^{n+1} \backslash\{0\}$ with coprime coordinates. This forms a hyperplane in the parameter space $\mathbf{Z}^{m}$, and contains $c(a) H^{m-1} / \phi(a)+O\left(H^{m-2}\right)$ integer points of height at most $H$, where $c(a)$ is the $(m-1)$-dimensional volume of the part of the hyperplane inside $[-1,1]^{m}$, and $\phi(a)$ is the covolume of the lattice of integer points lying on the hyperplane. Lemma 3.1 below shows that $\phi(a)$ equals the norm of the vector $b$ formed by the monomials of degree $d$ in the coordinates of $a$. If we ignore the error term, then we get that $N(H) \leq H^{m-1} \sum_{a} c(a) / \phi(a)$, where $a$ ranges over $\mathbf{Z}^{n+1} \backslash\{0\}$. Now $c(a)$ is bounded, and it is easy to show that $\sum 1 / \phi(a)$ converges precisely when $d>n+1$, so our heuristic predicts $N(H)=O\left(H^{m-1}\right)$. Since $N_{\text {tot }}(H) \sim(2 H)^{m}$, this leads to the first part of the conjecture.

Lemma 3.1. Let $b$ be a vector in $\mathbf{R}^{m}$ with coprime integer coordinates and norm $|b|=M$. The covolume of the lattice $\Lambda=\left\{x \in \mathbf{Z}^{m}:\langle x, b\rangle=0\right\}$ is $\sqrt{M}$.

Proof. The lattice $\Gamma=\left\{x \in \mathbf{Z}^{m}:\langle x, b\rangle \equiv 0(\bmod M)\right\}$ is the inverse image of $M \mathbf{Z}$ under the surjection $\mathbf{Z}^{m} \rightarrow \mathbf{Z}$ mapping $x$ to $\langle x, b\rangle$, so $\Gamma$ has covolume $M$ in $\mathbf{R}^{m}$. On the other hand, $\Gamma$ is the orthogonal direct sum of $\Lambda$ and $\mathbf{Z} b$, so the covolume of $\Lambda$ is $M /|b|=\sqrt{M}$.

Our next proposition will be conditional on cases of the following very general conjecture.

Conjecture 3.2 (Colliot-Thélène). Let $X$ be a smooth, proper, geometrically integral variety over a number field $k$. Suppose that $X$ is (geometrically) rationally connected. Then the Brauer-Manin obstruction to the Hasse principle for $X$ is the only obstruction.

Remark 3.3. Conjecture 3.2 has a long history. In the special case of rational surfaces, it appeared as Question (k1) on page 233 of [CTS80] (a paper later developed as [CTS87]), based on evidence eventually published in the papers [CTCS80] and [CTS82]. Theoretical evidence and some numerical evidence have been gathered since then, in the case of rational surfaces. The conjecture was generalized to (geometrically) unirational and Fano varieties on the first page of [CTSD94]. The full version of Conjecture 3.2 was raised as a question in lectures by Colliot-Thélène at the Institut Henri Poincaré in Spring 1999, and was repeated in print on page 3 of [CT03].

Proposition 3.4. Assume Conjecture 3.2. If $2 \leq d \leq n$, then $\left(N_{\mathrm{loc}}-N(H)\right) / N_{\text {tot }}(H) \rightarrow 0$ as $H \rightarrow \infty$. 
Proof. By Remark 2.1, we may restrict attention to $f$ 's for which $f=0$ defines a smooth, geometrically integral hypersurface $X$ in $\mathbf{P}^{n}$. The assumption $d \leq n$ implies that $X$ is Fano, hence rationally connected (see Theorem V.2.13 of [Kol96]). If $n \geq 4$, then by Corollary 4.2 there is no Brauer-Manin obstruction, so Conjecture 3.2 gives the Hasse principle, as desired. If $d=2$, then the Hasse principle holds unconditionally.

It remains to consider the case of cubic surfaces $(d=n=3)$. Here the Hasse principle does not always hold. But by [SD93] there is no Brauer-Manin obstruction whenever whenever the action of $\mathrm{Gal}(\overline{\mathbf{Q}} / \mathbf{Q})$ on the 27 lines is as large as possible (namely, the Weyl group $\left.W\left(E_{6}\right)\right)$. The Galois action on the 27 lines on the generic cubic surface over $\mathbf{Q}\left(a_{1}, \ldots, a_{20}\right)$ is $W\left(E_{6}\right)$ (this follows from [Tod35] and [Seg42]), so it follows by Hilbert irreducibility (see $\S 9.2$ and $\S 13$ of [Ser97]) that the same holds for a density 1 set of cubic surfaces over $\mathbf{Q}$. Such cubic surfaces, under Conjecture 3.2, satisfy the Hasse principle as desired.

Remark 3.5. For $n$ large compared to $d$, the conclusion of Proposition 3.4 can be proved unconditionally by using the circle method.

Part (ii) of Conjecture 2.2 would follow from the conclusion of Proposition 3.4 and the following result.

Theorem 3.6. If $n, d \geq 2$ and $(n, d) \neq(2,2)$ then $N_{\text {loc }}(H) / N_{\text {tot }}(H) \rightarrow c$ for some $c>0$. Moreover, $c=\prod c_{v}$ where $c_{v}$ is as in Conjecture 2.2.

Proof. By Hensel's Lemma, a hypersurface $f=0$ will have a point in $\mathbf{Q}_{p}$ if its reduction modulo $p$ has a smooth point in $\mathbf{F}_{p}$. If $f$ is absolutely irreducible modulo $p$ and $p$ is sufficiently large (in terms of $n$ and $d$ ), then the existence of a smooth point in $\mathbf{F}_{p}$ is ensured by the Lang-Weil estimate [LW54]. Lemmas 20 and 21 of [PS99a] will now imply the theorem, provided that we can show that the space of reducible polynomials is of codimension at least 2 in the space of all polynomials. The lower bound on the codimension follows from the inequalities

$$
\left(\left(\begin{array}{c}
n+r \\
n
\end{array}\right)-1\right)+\left(\left(\begin{array}{c}
n+(d-r) \\
n
\end{array}\right)-1\right) \leq\left(\left(\begin{array}{c}
n+d \\
n
\end{array}\right)-1\right)-2
$$

for $0<r<d$, which hold provided that $n, d \geq 2$ and $(n, d) \neq(2,2)$. (Here $r$ and $d-r$ represent degrees in a potential factorization.) See also [PS99b] for an exposition of the application of the density lemmas from [PS99a].

Remark 3.7. It follows from Theorem 3.6 and part (i) of Conjecture 2.2 that for each pair $(d, n)$ with $n \geq 2$ and $d>n+1$, there are hypersurfaces of degree $d$ in $\mathbf{P}^{n}$ for which the Hasse principle fails. There does not seem to be an unconditional proof of this statement yet, when $n \geq 3$. For results conditional on various conjectures see [SW95] and [Poo01].

\section{Appendix A by Jean-Louis Colliot-Thélène: The Brauer-Manin OBSTRUCTION FOR COMPLETE INTERSECTIONS OF DIMENSION $\geq 3$}

It seems that a full proof of the following proposition has never before appeared in print, though a sketch can be found in $\S 2$ of [SW95]. Let $\mathrm{H}^{i}$ below denote étale cohomology (or profinite group cohomology) unless otherwise specified, and let $\operatorname{Br} X$ denote the cohomological Brauer group $\mathrm{H}^{2}\left(X, \mathbf{G}_{m}\right)$ of a scheme $X$.

Proposition 4.1. Let $k$ be a field of characteristic 0 . Let $X$ be a smooth complete intersection in $\mathbf{P}_{k}^{n}$ satisfying $\operatorname{dim} X \geq 3$. Then the natural map $\operatorname{Br} k \rightarrow \operatorname{Br} X$ is an isomorphism. 
Proof. Let $\bar{k}$ denote an algebraic closure of $k$, let $G=\operatorname{Gal}(\bar{k} / k)$, and let $\bar{X}=X \times_{k} \bar{k}$. Let $p: X \rightarrow$ Spec $k$ denote the structure map. In the Leray spectral sequence

$$
\left.E_{2}^{p, q}:=\mathrm{H}^{p}\left(k, \mathrm{R}^{q} p_{*} \mathbf{G}_{m}\right)\right) \Longrightarrow E^{p+q}:=\mathrm{H}^{p+q}\left(X, \mathbf{G}_{m}\right),
$$

the étale sheaf $\mathrm{R}^{q} p_{*} \mathbf{G}_{m}$ on Spec $k$ corresponds to the $G$-module $\mathrm{H}^{q}\left(\bar{X}, \mathbf{G}_{m}\right)$. A smooth complete intersection of positive dimension is geometrically connected [Har77, Exercise III.5.5(b)], so $\mathrm{H}^{0}\left(\bar{X}, \mathbf{G}_{m}\right)=\bar{k}^{\times}$, and [BLR90, p. 203] shows that $\mathrm{H}^{1}\left(\bar{X}, \mathbf{G}_{m}\right)=\mathrm{H}_{\text {Zariski }}^{1}\left(\bar{X}, \mathbf{G}_{m}\right)=$ : Pic $\bar{X}$. Thus the exact sequence

$$
E_{2}^{1,0} \rightarrow E^{1} \rightarrow E_{2}^{0,1} \rightarrow E_{2}^{2,0} \rightarrow \operatorname{ker}\left(E^{2} \rightarrow E_{2}^{0,2}\right) \rightarrow E_{2}^{1,1}
$$

from the spectral sequence becomes

$$
0 \rightarrow \operatorname{Pic} X \rightarrow(\operatorname{Pic} \bar{X})^{G} \rightarrow \operatorname{Br} k \rightarrow \operatorname{ker}(\operatorname{Br} X \rightarrow \operatorname{Br} \bar{X}) \rightarrow \mathrm{H}^{1}(k, \operatorname{Pic} \bar{X}) .
$$

It remains to prove that $\operatorname{Pic} X \rightarrow(\operatorname{Pic} \bar{X})^{G}$ is an isomorphism, that $\mathrm{H}^{1}(k, \operatorname{Pic} \bar{X})=0$, and that $\operatorname{Br} \bar{X}=0$.

For smooth complete intersections of dimension $\geq 3$ in $\mathbf{P}^{n}, \mathbf{M}$. Noether proved that the restriction map Pic $\overline{\mathbf{P}^{n}} \rightarrow \operatorname{Pic} \bar{X}$ is an isomorphism (see Corollary 3.3 on p. 180 of [Har70] for a modern proof). The commutative square

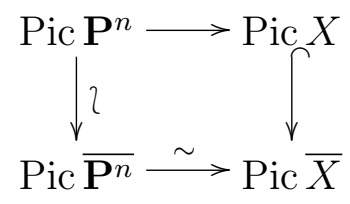

shows that the injections

$$
\operatorname{Pic} X \hookrightarrow(\operatorname{Pic} \bar{X})^{G} \hookrightarrow \operatorname{Pic} \bar{X}
$$

are isomorphisms, and that $\mathrm{H}^{1}(k, \operatorname{Pic} \bar{X})=\mathrm{H}^{1}(G, \mathbf{Z})=\operatorname{Hom}_{\text {conts }}(G, \mathbf{Z})=0$.

Finally we need to show that if $Y$ is a complete intersection of dimension $\geq 3$ in $\mathbf{P}^{n}$ over an algebraically closed field $L$ of characteristic 0 , then $\operatorname{Br} Y=0$. For each prime $\ell$, the Kummer sequence yields the exact rows of the diagram

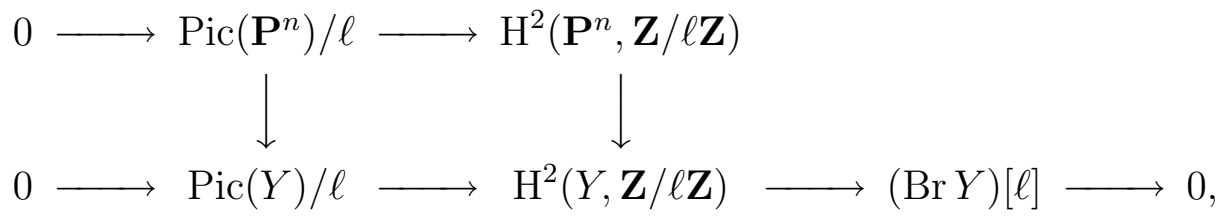

where for any abelian group $A$, the notation $A / \ell$ denotes $A / \ell A$, and $A[\ell]$ is the kernel of multiplication-by- $\ell$ on $A$. The top horizontal injection $\operatorname{Pic}\left(\mathbf{P}^{n}\right) / \ell \rightarrow \mathrm{H}^{2}\left(\mathbf{P}^{n}, \mathbf{Z} / \ell \mathbf{Z}\right)$ is an isomorphism since both groups are of rank 1 over $\mathbf{Z} / \ell \mathbf{Z}$. The right vertical map $\mathrm{H}^{2}\left(\mathbf{P}^{n}, \mathbf{Z} / \ell \mathbf{Z}\right) \rightarrow$ $\mathrm{H}^{2}(Y, \mathbf{Z} / \ell \mathbf{Z})$ is an isomorphism by a version of the Weak Lefschetz Theorem: see Corollary 5.6 in Appendix B of this paper. The diagram then implies that $(\mathrm{Br} Y)[\ell]=0$. This holds for all $\ell$, and $\operatorname{Br} Y$ is torsion [Gro68, Proposition 1.4], so $\operatorname{Br} Y=0$.

Corollary 4.2. If in addition, $k$ is a number field, then the Brauer-Manin obstruction for $X$ is vacuous.

Proof. This follows from Proposition 4.1, since the elements of $\operatorname{Br} X$ coming from $\operatorname{Br} k$ do not give any obstruction to rational points. 


\section{Appendix B by Nicholas M. Katz: Applications of the Weak Lefschetz THEOREM}

We work over an algebraically closed field $k$. Take as ambient space any separated $V / k$ of finite type which is smooth, and everywhere of dimension $N$ (i.e., each connected component of $V$ has the same dimension $N$ ). In $V$, we are given a certain number $r \geq 1$ of closed subschemes $H_{i}$, each of which has the property that its complement $V-H_{i}$ is affine. Define the closed subscheme $X$ of $V$ to be the intersection of the $H_{i}$. Its complement $V-X$ is covered by $r$ affine open sets, each of dimension (at most) $N$, namely the $V-H_{i}$.

Lemma 5.1. The scheme $V-X$ has cohomological dimension at most $N+r-1$, i.e., for any constructible torsion sheaf $\mathcal{F}$ on $V-X$, we have $\mathrm{H}^{i}(V-X, \mathcal{F})=0$ for $i \geq N+r$.

This is a special case of

Lemma 5.2. If a separated $k$-scheme $W / k$ of finite type is the union of $r$ affine opens $U_{i}$, each of dimension at most $N$, then $W$ has cohomological dimension at most $N+r-1$.

Proof. For $r=1$, this is just the Lefschetz affine theorem [SGA4 III, Exposé XIV, Corollaire 3.2]. For general $r$, one proceeds by induction on $r$, writing $W$ as the union of the two open sets $A:=U_{r}$ and $B:=\bigcup_{i<r} U_{i}$. Then $A \cap B$ is the union of $r-1$ affines, and we use the long exact sequence

$$
\ldots \rightarrow \mathrm{H}^{i}(A \cup B, \mathcal{F}) \rightarrow \mathrm{H}^{i}(A, \mathcal{F}) \oplus \mathrm{H}^{i}(B, \mathcal{F}) \rightarrow \mathrm{H}^{i}(A \cap B, \mathcal{F}) \rightarrow \ldots
$$

to get the assertion.

Suppose now that $\ell$ is a prime number invertible in the field $k$, and that $\mathcal{F}$ is a $\mathbf{Z} / \ell \mathbf{Z}$ sheaf on $V$ whose restriction to $V-X$ is lisse, for instance $\mathbf{Z} / \ell \mathbf{Z}$ itself. Because $V-X$ is smooth, everywhere of dimension $N$, the Poincare dual of Lemma 5.1 is the vanishing of compact cohomology up through dimension $N-r$ :

Lemma 5.3. For any integer $i \leq N-r$, we have $\mathrm{H}_{c}^{i}(V-X, \mathcal{F})=0$.

Now use the excision sequence (this is why we need $\mathcal{F}$ to be a sheaf on $V$ ) in compact cohomology

$$
\ldots \rightarrow \mathrm{H}_{c}^{i}(V-X, \mathcal{F}) \rightarrow \mathrm{H}_{c}^{i}(V, \mathcal{F}) \rightarrow \mathrm{H}_{c}^{i}(X, \mathcal{F}) \rightarrow \ldots
$$

to conclude

Theorem 5.4. For any integer $i<N-r$, the restriction map

$$
\mathrm{H}_{c}^{i}(V, \mathcal{F}) \rightarrow \mathrm{H}_{c}^{i}(X, \mathcal{F})
$$

is an isomorphism. For $i=N-r$, this map is injective.

Corollary 5.5. If $V / k$ is in addition assumed proper, then we have the same result for non-compact cohomology: For any integer $i<N-r$, the restriction map

$$
\mathrm{H}^{i}(V, \mathcal{F}) \rightarrow \mathrm{H}^{i}(X, \mathcal{F})
$$

is an isomorphism. For $i=N-r$, this map is injective.

As a special case of Corollary 5.5, we get

Corollary 5.6. Suppose $X$ is a closed subscheme of projective space $\mathbf{P}^{N}$ which is defined by the vanishing of $N-d$ homogeneous forms. Then for $i<d$, the restriction map

$$
\mathrm{H}^{i}\left(\mathbf{P}^{N}, \mathbf{Z} / \ell \mathbf{Z}\right) \rightarrow \mathrm{H}^{i}(X, \mathbf{Z} / \ell \mathbf{Z})
$$

is an isomorphism. For $i=d$, this map is injective. 
Remark 5.7. In Corollary 5.6, it is enough if $X$ is defined set-theoretically by the vanishing of $N-d$ homogeneous forms, since $X$ and $X^{\text {red }}$ have the same étale cohomology.

\section{ACKNOWLEDGEMENTS}

This paper developed during a workshop sponsored by the American Institute of Mathematics. We thank Jean-Louis Colliot-Thélène and Nick Katz for allowing us to include their results in the appendices to this paper. We thank also Jean-Louis Colliot-Thélène, Jordan Ellenberg, Brendan Hassett, Roger Heath-Brown, Bill McCallum, and Trevor Wooley for many conversations which helped shape Conjecture 2.2.

\section{REFERENCES}

[BLR90] Siegfried Bosch, Werner Lütkebohmert, and Michel Raynaud, Néron models, Springer-Verlag, Berlin, 1990.

[CT03] Jean-Louis Colliot-Thélène, Points rationnels sur les fibrations, Higher dimensional varieties and rational points, Proceedings of the 2001 Budapest conference (K. Böröczky, J. Kollár, and T. Szamuely, eds.), Springer-Verlag, 2003, Bolyai Society Colloquium Publications.

[CTCS80] Jean-Louis Colliot-Thélène, Daniel Coray, and Jean-Jacques Sansuc, Descente et principe de Hasse pour certaines variétés rationnelles, J. Reine Angew. Math. 320 (1980), 150-191.

[CTS80] J.-L. Colliot-Thélène and J.-J. Sansuc, La descente sur les variétés rationnelles, Journées de Géometrie Algébrique d'Angers, Juillet 1979/Algebraic Geometry, Angers, 1979, Sijthoff \& Noordhoff, Alphen aan den Rijn, 1980, pp. 223-237.

[CTS82] Jean-Louis Colliot-Thélène and Jean-Jacques Sansuc, Sur le principe de Hasse et l'approximation faible, et sur une hypothèse de Schinzel, Acta Arith. 41 (1982), no. 1, 33-53.

[CTS87] Jean-Louis Colliot-Thélène and Jean-Jacques Sansuc, La descente sur les variétés rationnelles. II, Duke Math. J. 54 (1987), no. 2, 375-492.

[CTSD94] Jean-Louis Colliot-Thélène and Peter Swinnerton-Dyer, Hasse principle and weak approximation for pencils of Severi-Brauer and similar varieties, J. Reine Angew. Math. 453 (1994), 49-112.

[Gro68] Alexander Grothendieck, Le groupe de Brauer. II. Théorie cohomologique, Dix Exposés sur la Cohomologie des Schémas, North-Holland, Amsterdam, 1968, pp. 67-87.

[Har70] Robin Hartshorne, Ample subvarieties of algebraic varieties, Notes written in collaboration with C. Musili. Lecture Notes in Mathematics, Vol. 156, Springer-Verlag, Berlin, 1970.

[Har77] Robin Hartshorne, Algebraic geometry, Springer-Verlag, New York, 1977, Graduate Texts in Mathematics, No. 52.

[Kol96] János Kollár, Rational curves on algebraic varieties, Ergebnisse der Mathematik und ihrer Grenzgebiete. 3. Folge. A Series of Modern Surveys in Mathematics [Results in Mathematics and Related Areas. 3rd Series. A Series of Modern Surveys in Mathematics], vol. 32, Springer-Verlag, Berlin, 1996.

[LW54] Serge Lang and André Weil, Number of points of varieties in finite fields, Amer. J. Math. 76 (1954), 819-827.

[Poo01] Bjorn Poonen, The Hasse principle for complete intersections in projective space, Rational points on algebraic varieties, Progr. Math., vol. 199, Birkhäuser, Basel, 2001, pp. 307-311.

[PS99a] Bjorn Poonen and Michael Stoll, The Cassels-Tate pairing on polarized abelian varieties, Ann. of Math. (2) 150 (1999), no. 3, 1109-1149.

[PS99b] Bjorn Poonen and Michael Stoll, A local-global principle for densities, Topics in number theory (University Park, PA, 1997), Math. Appl., vol. 467, Kluwer Acad. Publ., Dordrecht, 1999, pp. 241-244.

[SD93] Peter Swinnerton-Dyer, The Brauer group of cubic surfaces, Math. Proc. Cambridge Philos. Soc. 113 (1993), no. 3, 449-460.

[Seg42] B. Segre, The Non-singular Cubic Surfaces, Oxford University Press, Oxford, 1942. 
[Ser97] Jean-Pierre Serre, Lectures on the Mordell-Weil theorem, third ed., Friedr. Vieweg \& Sohn, Braunschweig, 1997, Translated from the French and edited by Martin Brown from notes by Michel Waldschmidt, With a foreword by Brown and Serre.

[SGA4 III] Théorie des topos et cohomologie étale des schémas. Tome 3, Springer-Verlag, Berlin, 1973, Séminaire de Géométrie Algébrique du Bois-Marie 1963-1964 (SGA 4), Dirigé par M. Artin, A. Grothendieck et J. L. Verdier. Avec la collaboration de P. Deligne et B. Saint-Donat, Lecture Notes in Mathematics, Vol. 305.

[SW95] Peter Sarnak and Lan Wang, Some hypersurfaces in $\mathbf{P}^{4}$ and the Hasse-principle, C. R. Acad. Sci. Paris Sér. I Math. 321 (1995), no. 3, 319-322.

[Tod35] J. A. Todd, On the topology of certain threefold loci, Proc. Edinburgh Math. Soc. (2) (1935), no. $4,175-184$.

Department of Mathematics, University of California, Berkeley, CA 94720-3840, USA

E-mail address: poonen@math.berkeley.edu

Department of Mathematics, University of Texas, Austin, TX 78712, USA

E-mail address: voloch@math.utexas.edu 